\title{
Prognostic and predictive enrichment in sepsis
}

\author{
Natalja L. Stanski ${ }^{1}$ and Hector R. Wong $\mathbb{1}^{1,2 *}$
}

Abstract | Sepsis is a heterogeneous disease state that is both common and consequential in critically ill patients. Unfortunately, the heterogeneity of sepsis at the individual patient level has hindered advances in the field beyond the current therapeutic standards, which consist of supportive care and antibiotics. This complexity has prompted attempts to develop a precision medicine approach, with research aimed towards stratifying patients into more homogeneous cohorts with shared biological features, potentially facilitating the identification of new therapies. Several investigators have successfully utilized leukocyte-derived mRNA and discovery-based approaches to subgroup patients on the basis of biological similarities defined by transcriptomic signatures. A critical next step is to develop a consensus sepsis subclassification system, which includes transcriptomic signatures as well as other biological and clinical data. This goal will require collaboration among various investigative groups, and validation in both existing data sets and prospective studies. Such studies are required to bring precision medicine to the bedside of critically ill patients with sepsis.

Sepsis can be broadly defined as a systemic response to a microbial infection and is a common diagnosis among critically ill patients. Research insights, as well as failed attempts to identify sepsis-specific disease-modifying therapies, have highlighted the incredible variability of this syndrome at the individual patient level ${ }^{1}$. Appropriately, the current consensus definition of sepsis - "a lifethreatening organ dysfunction caused by a dysregulated host response to infection" - is broad, reflecting the intrinsic heterogeneity of the disease ${ }^{2}$. The source of this heterogeneity is multifaceted: infection aetiologies vary, individual host comorbidities and genetics are unique, and the timeliness of diagnosis and treatment differ from patient to patient ${ }^{1,3}$. These factors not only impact the evolution of sepsis at the individual patient level but also their responses to a therapeutic intervention.

Unfortunately, despite a growing understanding of the variability in the host immune response to sepsis, the field has not advanced much beyond the mainstays of prevention, early recognition and supportive care ${ }^{1,3}$. This failure is not due to a lack of dedicated research efforts, as well over 100 clinical trials have tested therapies that modify the systemic inflammatory response, all without significant positive results ${ }^{3}$. Recognition of the heterogeneity of sepsis, coupled with the failure to identify effective therapies, have driven attempts to establish a precision medicine approach to the diagnosis and treatment of sepsis.

In this Review, we outline the attempts that have been made thus far to resolve the heterogeneity of sepsis and propose next steps to advance the field. We also reflect on how principles learned from efforts to develop precision medicine in sepsis might be applied to the prediction and understanding of the pathogenesis of sepsisassociated acute kidney injury (AKI). Considerable progress has been made over the past decade, but collaborations between investigators and industry are still required to enable the application of a precision medicine strategy at the bedside of a critically ill patient with sepsis.

\section{Precision medicine and enrichment}

Precision medicine refers to prevention, diagnostic and treatment strategies that take individual patient characteristics into account ${ }^{4,5}$. This approach was conceptualized and is currently most advanced in the field on oncology, as genetic and molecular tumour markers have been identified and successfully targeted with tailored therapeutic agents ${ }^{6-8}$. In the USA, recognition of the breakthroughs facilitated by utilizing this approach have led to the creation of the 'Precision Medicine Initiative' by the federal government in 2015 . This initiative includes the expansion of precision medicine clinical trials and the provision of incentives for industry to develop more efficient and novel genomic sequencing techniques, among other efforts ${ }^{4,9}$. Although much of this initiative continues to focus on cancer, the application of precision medicine principles to other heterogeneous disease processes, such as sepsis, is a logical next step and an area of growing research interest. 


\section{Key points}

- Sepsis is a heterogeneous clinical syndrome and its variability at the individual patient level makes it amenable to a precision medicine approach for prevention, diagnosis and treatment.

- The concept of enrichment - including both prognostic and predictive enrichment - is fundamental to enabling precision medicine.

- Several investigators have utilized leukocyte-derived mRNA and a discovery-based approach to identify sepsis subclassification systems for both prognostic and predictive enrichment.

- An ideal approach would utilize enrichment strategies for clinical trial design and bedside decision-making in real time, but considerable work is still required to reach clinical feasibility.

- Understanding the sepsis molecular signature of a patient will likely be crucial to improving the treatment of sepsis-associated acute kidney injury, which is a common complication associated with substantial morbidity and mortality.

Machine learning A form of artificial intelligence wherein algorithms build a mathematical model using sample data to make predictions or decisions.

Latent class analysis A form of mathematical modelling used to find subtypes of cases using multivariate categorical data

Septic shock

Sepsis combined with cardiovascular failure.
One of the key tenets of precision medicine is the concept of enrichment ${ }^{5,10}$. Generally, enrichment refers to the selection of a subgroup of patients who are more likely to respond to a given therapy, when compared with an unselected population ${ }^{11}$. Enrichment strategies can be further categorized as prognostic or predictive. Prognostic enrichment refers to the selection of patients with a higher likelihood of having a disease-related outcome of interest, such as mortality ${ }^{5,10,11}$. Employment of this strategy is particularly important when considering the risk-to-benefit ratio of a potential therapy, as therapies carrying more than minimal risk might only be justified in patients with a higher likelihood of a diseaserelated event ${ }^{5}$. Additionally, the use of prognostic enrichment strategies is an important tool in study design, as preferentially selecting patients with higher event rates can both improve the power of the study and reduce the number of patients needed for enrolment ${ }^{11}$. A pertinent example of this concept is the CONSENSUS trial, which demonstrated in a relatively small sample size $(n=253)$ that the ACE inhibitor enalapril significantly reduced mortality in patients with severe congestive heart failure, whereas subsequent similar studies that included patients with varying illness severity required thousands of patients to demonstrate a drug benefit ${ }^{12}$.

By contrast, predictive enrichment involves selecting patients who are more likely to respond to a given therapy on the basis of a biological mechanism ${ }^{5,10,11}$. A classic example of this type of enrichment is demonstrated by the successful use of trastuzumab, a humanized monoclonal antibody targeting the gene HER2 (also known as transmembrane receptor tyrosine-protein kinase erbB2), in patients with HER2-positive breast cancer ${ }^{13}$. However, predictive enrichment requires a clear understanding of the biological mechanisms underlying the disease of interest, often making these strategies more challenging to develop and employ than prognostic enrichment strategies. This is particularly true in the context of sepsis, wherein there is no unifying targetable biological mechanism analogous to that described above for HER2-positive breast cancer.

Optimal advancement of precision medicine for sepsis will require parallel application of both prognostic and predictive enrichment (FIG. 1). Specifically, patients are initially risk-stratified utilizing prognostic enrichment and low-risk patients are allocated to standard care. Those patients at a higher risk of a poor outcome can then be further separated on the basis of biological commonalities using a predictive enrichment strategy to identify unique subgroups of patients who might benefit from targeted adjunctive therapies ${ }^{5,11}$. This combination approach aligns with current guidelines proposed by the $\mathrm{FDA}^{11}$. As the field of precision medicine continues to expand in its application including rapidly evolving critical illnesses such as sepsis - progress towards this goal will rely on developing our understanding of the underlying mechanisms of disease and technological advances that enable real-time enrichment to occur at the bedside.

\section{Enrichment in sepsis}

Efforts to develop enrichment strategies for sepsis have utilized both clinical parameters and the current understanding of biological mechanisms. Clinically, different sepsis 'subphenotypes' have been proposed based on shared clinical features and using tools such as machine learning and latent class analysis ${ }^{14-17}$. Similarly, advances in the field of genomics have allowed several investigators to utilize leukocyte-derived RNA to identify cohorts of patients with shared biological features at the gene expression level ${ }^{18-31}$. The ability to identify these different patient cohorts has enabled the development of both prognostic and predictive enrichment strategies for sepsis.

Prognostic enrichment in sepsis. An example of prognostic enrichment in sepsis is the Paediatric Sepsis Biomarker Risk Model (PERSEVERE) ${ }^{18}$. The initial model incorporated five serum protein biomarkers C-C chemokine ligand 3 (CCL3), IL-8, granzyme B, heat shock protein $70 \mathrm{kDa} 1 \mathrm{~B}$ and matrix metallopeptidase 8 (MMP8; also known as neutrophil collagenase) originally identified using transcriptomics to select candidate genes associated with poor outcome in a population of paediatric patients with septic shock ${ }^{32}$. From approximately 80 candidate genes identified, we subsequently reduced the list to 12 genes based on two a priori criteria. The first criterion was biological plausibility linking the gene to sepsis biology, based on existing knowledge. The second criterion was the ability to measure the protein product of the gene in serum. These 12 initial PERSEVERE biomarkers were subsequently reduced to five biomarkers using classification and regression tree (CART) methodology to develop a model that estimated the risk of 28-day mortality among children with septic shock (PERSEVERE). This approach provided a potential strategy for prognostic enrichment that could inform clinical decision-making and trial design ${ }^{18,32}$. Since its inception, this model has been prospectively validated and recalibrated as PERSEVERE-II (which includes platelet count in addition to the original biomarkers) ${ }^{19,20}$ and has been used to design a comparable model for adult patients with septic shock $^{21}$. Most recently, PERSEVERE-II was remodelled to include mRNA biomarkers (PERSEVERE-XP), further increasing its predictive performance ${ }^{22}$.

One research group utilized a discovery approach to prognostic enrichment and combining clinical features 
Group-based trajectory modelling

A statistical method used to identify groups of individuals who follow a similar trajectory over time (serum lactate concentrations, age and haematocrit) with metabolomic data (carnitine ester levels) to create a prediction model for sepsis survival. This work highlighted the potential role of decreased fatty acid oxidation in sepsis non-survivors ${ }^{29}$. In another study, investigators demonstrated that a coding variant of the IL-1 receptor antagonist gene (rs315952) was associated with improved survival and faster resolution of shock in the Vasopressin and Septic Shock Trial cohort ${ }^{33}$. This coding variant was associated with higher levels of IL-1 receptor antagonist both at baseline and in response to a human endotoxin challenge ${ }^{33}$, suggesting that plasma IL-1 receptor antagonist has a protective effect in sepsis.

On a larger scale, one group of researchers utilized all publicly available transcriptomic data for patients with sepsis (including adults, children and neonates) to develop mortality prediction models ${ }^{30}$. In this study, machine-learning approaches were used for gene identification and four different prediction models were independently developed and validated; their predictive performance was further improved when combined with scores of illness severity ${ }^{30}$. Interestingly, of the 65 candidate mortality predictor genes identified in these four models, 11 overlapped with the initial 80 genes identified in the PERSEVERE discovery phase $\mathrm{e}^{22,32}$. These 11 genes have biological links to tumour necrosis factor (TNF), cellular tumour antigen p53 and IL-8, all of which have been previously linked to the pathobiology of sepsis (FIG. 2). The identification of these candidate genes of interest by multiple investigators further supports the hypothesis that they contribute to the mechanisms underlying poor outcomes in sepsis and thereby also represent potential therapeutic targets.

Investigators have also used clinical data alone to subphenotype sepsis for the purposes of prognostic enrichment. One research group utilized latent class analysis of over 14,000 patients with sepsis to identify four subclasses of patients with differing mortality rates: baseline type (profile 1, lowest mortality), respiratory dysfunction type (profile 2), multiple organ dysfunction type (profile 3 , highest mortality) and neurological dysfunction type (profile 4$)^{14}$. A similar study examined the temperature trends of patients with sepsis and, through group-based trajectory modelling, delineated four different groups of patients with unique baseline mortality risks ${ }^{16}$. Their analysis identified 'hyperthermic, slow resolvers', 'hyperthermic, fast resolvers', 'normothermic' and 'hypothermic' subgroups of patients. The 'hypothermic' group had the highest mortality risk and the lowest levels of inflammatory markers measured ${ }^{16}$. A subsequent study utilizing a machine-learning strategy identified four sepsis phenotypes predictive of varying mortality risk based on a variety of clinical parameters, such as the presence or absence of renal or hepatic dysfunction, age and vasopressor requirement ${ }^{17}$. Although not directly based on biological mechanisms, these proposed clinical subphenotypes might provide a pragmatic and feasible approach to risk-stratifying patients with sepsis at the bedside, potentially enabling prognostic enrichment.

Predictive enrichment in sepsis. As noted previously, the use of predictive enrichment strategies requires the understanding and recognition of shared biological features among subgroups of patients ${ }^{5}$. To date, perhaps the most well-developed predictive enrichment strategies are based on gene expression signatures. Unlike prognostic enrichment, the separation of patients for the purposes of predictive enrichment does not necessarily take into account patient demographic characteristics, clinical course or outcomes. Instead, these strategies seek to group patients primarily on the basis of biological commonalities with the overarching goal of identifying patients likely to respond to a given therapeutic intervention. Efforts by investigative teams to subgroup patients with sepsis based on gene expression signatures have led to the development of four major classification systems, which are outlined below.

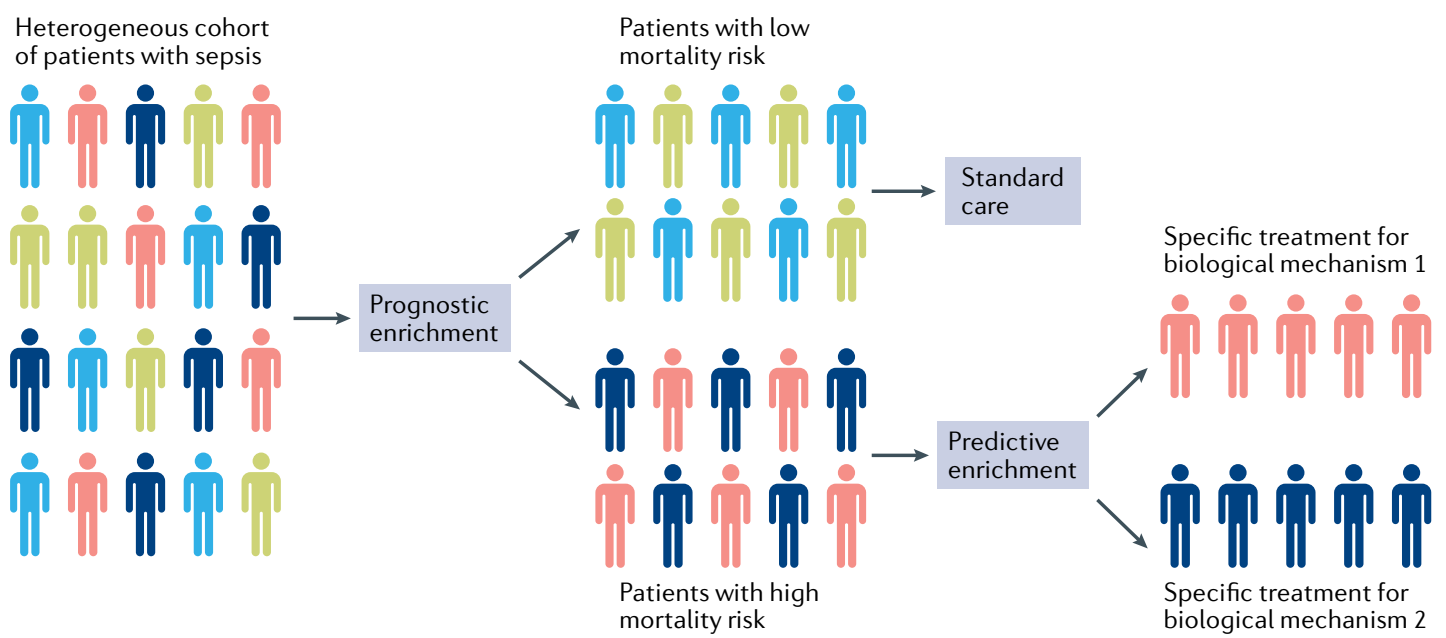

Fig. 1 Simplified diagram illustrating a generic approach to prognostic and predictive enrichment of sepsis. A heterogeneous cohort of patients with sepsis is first divided using prognostic enrichment into those at a low and those at a high risk of mortality. Patients who are at a low mortality risk are treated using standard care. The high-risk patients can be further divided using predictive enrichment and treated with targeted therapies based on their underlying, subgroup-defining biology. 
We reported the first system for gene expressionbased subgrouping of patients with sepsis in a study that involved children with septic shock; the patient subgroups were termed endotypes $\mathrm{A}$ and $\mathrm{B}^{25-28,34}$. Patients classified as endotype A had higher rates of mortality, even after correction for severity of illness, age and comorbidities; this was particularly true if allocation to endotype A persisted over the first $72 \mathrm{~h}$ of illness ${ }^{34}$. From a molecular standpoint, endotype A was characterized by repression of genes associated with adaptive immunity and glucocorticoid receptor signalling ${ }^{25}$. In line with this molecular signature, the prescription of

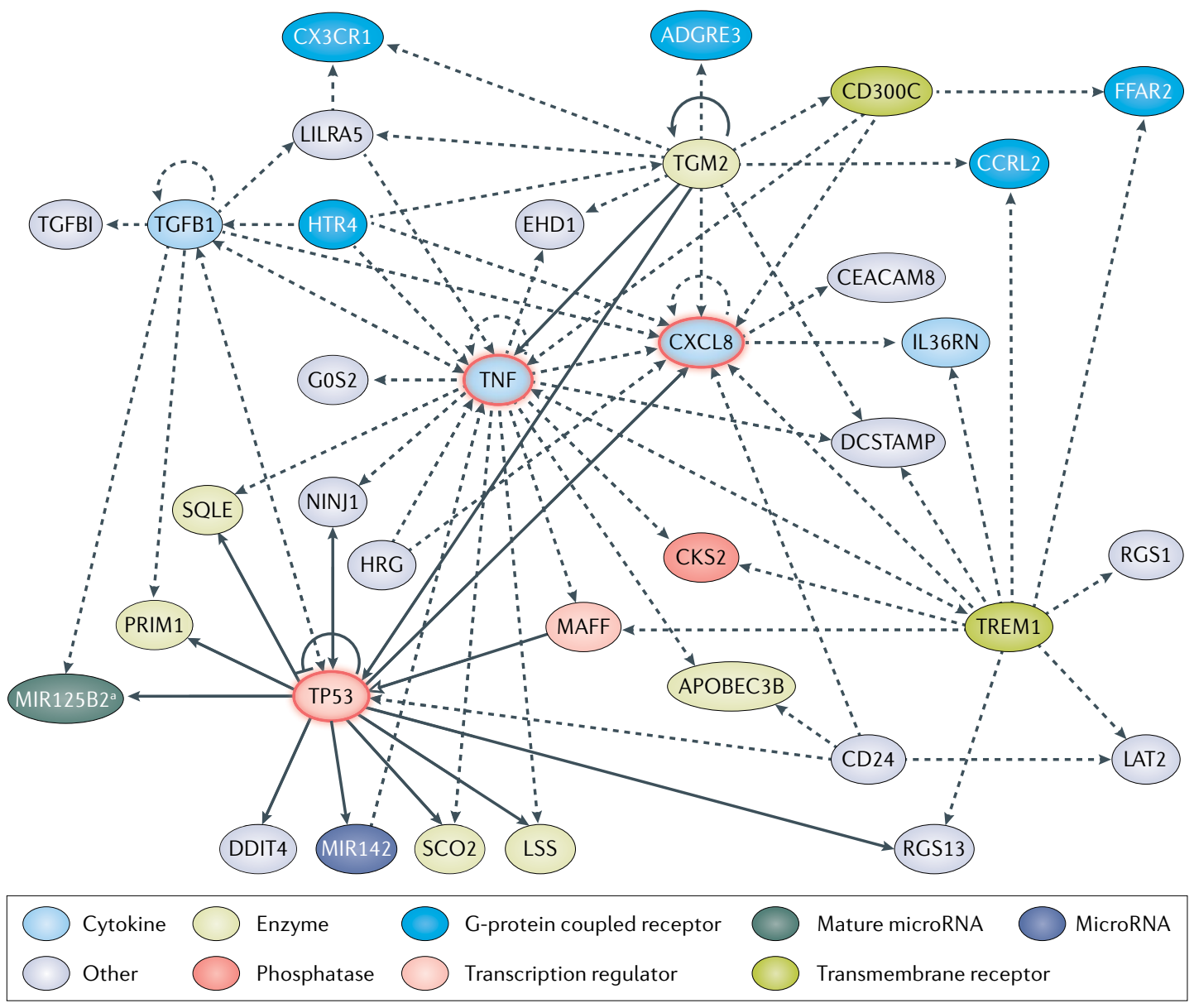

Fig. 2 | Network of candidate genes for predicting patient mortality identified through prognostic enrichment strategies. The community approach to mortality risk prediction in a sepsis ${ }^{30}$ study identified 65 risk-predicting genes based on publicly available gene expression data from adults, children and neonate with sepsis. Similarly, during the initial discovery phase of the PERSEVERE biomarkers ${ }^{32}$ used for prognostic enrichment, 80 genes associated with poor outcome were selected based on transcriptomic data from a cohort of paediatric patients with septic shock. An analysis of the genes identified in these two studies revealed 11 genes in common: adhesion G protein-coupled receptor E3 (ADGRE3), CD24 molecule (CD24), carcinoembryonic antigen-related cell adhesion molecule 8 (CEACAM8), CDC28 protein kinase regulatory subunit 2 (CKS2), C-X3-C motif chemokine receptor 1 (CX3CR1), DNA damage inducible transcript 4 (DDIT4), G0/G1 switch 2 (G0S2), C-X-C motif chemokine ligand 8 (CXCL8, also known as IL-8), MAF bZIP transcription factor $F$ (MAFF), regulator of $\mathrm{G}$ protein signalling 1 (RGS1) and transforming growth factor beta induced (TGFBI). The network was generated by uploading these 11 genes to the Ingenuity Pathway Analysis platform. Solid lines represent direct interactions and dashed lines represent indirect interactions; solid arrowheads can represent activation, modification and phosphorylation among others (for more details see Ingenuity Pathway Analysis legend), open arrowhead represents translocation and bar-headed arrow indicates that the gene inhibits and acts on its target. The gene network contains three highly connected central nodes relevant to the pathobiology of sepsis and inflammation: tumour necrosis factor (TNF), CXCL8 and tumour protein p53 (TP53). APOBEC3B, apolipoprotein B mRNA editing enzyme catalytic subunit 3B; CCRL2, C-C motif chemokine receptor like 2; CD300C, CMRF35-like molecule 6; DCSTAMP, dendrocyte expressed seven transmembrane protein; EHD1, EH domain containing 1; FFAR2, free fatty acid receptor 2; HRG, histidine-rich glycoprotein; HTR4, 5-hydroxytryptamine receptor 4; IL36RN, interleukin 36 receptor antagonist; LAT2, linker for activation of T cells family member 2; LILRA5, leukocyte immunoglobulin-like receptor A5; LSS, lanosterol synthase; MIR142, microRNA 142; NINJ1, ninjurin 1; PRIM1, DNA primase subunit 1; RGS13, regulator of G protein signalling 13; SCO2, SCO cytochrome c oxidase assembly protein 2; SQLE, squalene epoxidase; TGFB1, transforming growth factor beta

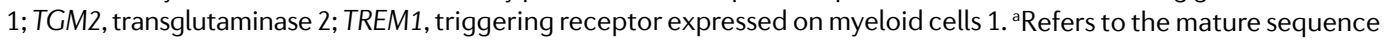
miR-125b-2-3p, encoded in the micro RNA-125b-2 (MIR125B2) stem loop sequence, and includes other microRNAs with the seed sequence CAAGUCA. 
Table 1 | Features of and overlap among gene expression-based sepsis subgroups

\begin{tabular}{|c|c|c|c|c|}
\hline Nomenclature & Biological features & Clinical association & Overlapping subgroups & Refs \\
\hline SRS1 and 2 & $\begin{array}{l}\text { SRS1 characterized by endotoxin } \\
\text { tolerance, T cell exhaustion and } \\
\text { downregulation of MHC class II }\end{array}$ & $\begin{array}{l}\uparrow \text { Mortality in SRS1; } \\
\text { corticosteroids associated } \\
\text { with } \uparrow \text { mortality in SRS2 }\end{array}$ & $\begin{array}{l}\text { - Endotype A and SRS1a } \\
\text { - SRS2 and Mars3 }\end{array}$ & 31,35 \\
\hline Mars1-4 & $\begin{array}{l}\text { Repression of genes } \\
\text { corresponding to innate and } \\
\text { adaptive immunity in Mars1 }\end{array}$ & $\uparrow$ Mortality in Mars1 & $\begin{array}{l}\text { - Mars3 and SRS2 } \\
\text { - Mars3 not detected } \\
\text { in children }\end{array}$ & 23 \\
\hline $\begin{array}{l}\text { Inflammopathic, } \\
\text { adaptive, } \\
\text { coagulopathic }\end{array}$ & $\begin{array}{l}\text { Differential expression of genes } \\
\text { associated with inflammation, } \\
\text { adaptive immunity or coagulation }\end{array}$ & $\begin{array}{l}\uparrow \text { Mortality in } \\
\text { coagulopathic subgroup }\end{array}$ & $\begin{array}{l}\text { - Inflammopathic and } \\
\text { SRS1 or endotype B } \\
\text { - Adaptive and SRS2 }\end{array}$ & 30 \\
\hline $\begin{array}{l}\text { Endotypes A } \\
\text { and B }\end{array}$ & $\begin{array}{l}\text { Repression of genes } \\
\text { corresponding to glucocorticoid } \\
\text { receptor signalling and adaptive } \\
\text { immunity in endotype A }\end{array}$ & $\begin{array}{l}\uparrow \text { Mortality in endotype A; } \\
\text { corticosteroids associated } \\
\text { with mortality in endotype A }\end{array}$ & $\begin{array}{l}\text { - SRS1 } 1^{\text {a }} \text { pathway } \\
\text { enrichment (adaptive } \\
\text { immunity) } \\
\text { - Age-dependent effects } \\
\text { on SRS and endotype } \\
\text { assignment }\end{array}$ & $25-28$ \\
\hline
\end{tabular}

Mars, molecular diagnosis and risk stratification of sepsis; MHC, major histocompatibility complex; SRS, sepsis response signatures. aPathways associated with adaptive immunity are enriched in both subgroups but overlap in gene expression pattern is minimal.

corticosteroids in patients assigned to endotype A was independently associated with increased risk of mortality $^{28}$. As the use of corticosteroids in the management of septic shock continues to be an unresolved topic of controversy, and the field is contemplating the use of therapeutic strategies to augment the adaptive immune response, these paediatric endotypes might represent a predictive enrichment strategy that enhances future clinical trial enrolment.

The Genomic Advances in Sepsis (GAinS) study examined adult patients with sepsis secondary to community-acquired pneumonia and identified two gene expression-based subgroups, sepsis response signatures (SRS) 1 and $2\left(\right.$ REF. $\left.^{31}\right)$. The SRS1 subgroup was characterized by an immunosuppressed phenotype that was associated with worse outcomes; its key features included genes linked to endotoxin tolerance, $\mathrm{T}$ cell exhaustion and downregulation of major histocompatibility complex class II. The presence of the SRS1 and 2 subgroups has since been prospectively validated in a follow-up study that included patients with sepsis caused by both pulmonary and abdominal infections $s^{35}$. The ability to identify a cohort of immunosuppressed patients offers an opportunity for predictive enrichment, as these patients might be more likely to benefit from therapies aimed at restoring the adaptive immune response. This investigative team also reported that corticosteroid prescription is associated with increased risk of mortality among patients assigned to the SRS2 subgroup ${ }^{36}$.

The Molecular Diagnosis and Risk Stratification of Sepsis study examined adult patients with sepsis and identified four distinct molecular subgroups, termed endotypes Mars1-4 (REF. ${ }^{23}$ ). Similar to the SRS1 group, the Mars1 endotype was associated with decreased expression of genes associated with innate and adaptive immune signalling, whereas the Mars3 endotype was characterized by an upregulation of adaptive immune pathways. Consistent with previous studies, patients classified as Mars1 had higher rates of mortality, whereas a Mars3 classification was associated with improved patient outcomes when compared with the other endotypes. Identifying patients who belong to the relatively immunosuppressed Mars1 cohort again provides an opportunity for predictive enrichment, as therapies to augment the immune system might be better directed towards these patients.

Finally, the Khatri laboratory utilized all publicly available transcriptomic data, which included data from adult and paediatric (children and neonates) patients with sepsis, to identify three gene expression-based clusters of patients, termed inflammopathic, adaptive and coagulopathic ${ }^{24}$. The gene expression pattern of the inflammopathic cluster suggested overactivation of the innate immune system and a relative lack of an adaptive immune response, whereas the gene expression pattern of the adaptive cluster suggested a high level of activation of the adaptive immune system. By contrast, the gene expression pattern of the coagulopathic cluster indicated overactivation of the coagulation cascade. These different cohorts had unique mortality risks highest in the inflammopathic and coagulopathic groups - but, perhaps more importantly, the identification of these biologically different subsets of patients also provides an opportunity for predictive enrichment.

Overlap in reported sepsis subgroups. Although the aforementioned gene expression-based classification systems for sepsis clearly share some commonalities with regard to gene expression patterns associated with inflammation and immunity (TABLE 1), whether they are describing the same subgroups remains unclear. Given the tendency towards poor outcome in both the SRS1 and paediatric endotype A groups, the GAinS investigators examined the potential overlap between these two different subsets of patients. Despite an overlap in pathway enrichment, including $\mathrm{T}$ cell and B cell signalling, the overlap in specific gene expression was minimal ${ }^{35}$. In another study, the adult patients from the GAinS cohort were assigned to paediatric endotypes $\mathrm{A}$ and $\mathrm{B}$ and this analysis demonstrated a relative lack of redundancy across the two classification schemes ${ }^{37}$. Conversely, the MARS investigators demonstrated a substantial overlap between their low-risk Mars3 subset and the SRS2 
subgroup $^{23}$, whereas the Khatri laboratory reported overlap between their inflammopathic cluster and SRS1, as well as between their adaptive cluster and SRS2 (REF. ${ }^{24}$ ). Further investigation of the similarities and differences between patient subgroups will be essential to achieve a harmonized consensus sepsis subgrouping system that can be applied to the clinic.

Impact of developmental age. In addition to the differences in study design and in the statistical approaches used to identify subgroup-defining genes, a potential major factor that complicates the harmonizing of different sepsis classification schemes is developmental age. Both experimental and clinical evidence demonstrate that age has an important role in regulating the host immune response to sepsis, with neonates and elderly adults in particular often exhibiting defects in innate and adaptive immunity that contribute to poor outcome ${ }^{38-44}$. The complex interaction between age and sepsis subgrouping was demonstrated in a study that attempted to assign paediatric endotypes $\mathrm{A}$ and $\mathrm{B}$ to patients from the adult GAinS cohort ${ }^{37}$. Although the proportions of adult patients assigned to endotypes $A$ and $B$ were similar to those observed in paediatric patients, the associated differences in mortality noted in the paediatric subgroups were not replicated in the adult cohort. However, when further stratified by age, there was a significant interaction among SRS assignment, endotype assignment and age. Specifically, younger patients co-assigned to SRS1 and endotype A had a substantially higher risk of mortality, compared with the other possible combinations of age, endotype and SRS assignment ${ }^{37}$. In addition, adults co-assigned to SRS1 and endotype A had the highest rate of mortality, compared with the other three possible co-assignment groups. Further study on the impact of developmental age on the host immune response to sepsis is necessary. The aforementioned observations suggest that any attempts to unify the different sepsis subgroups for the purposes of prognostic and predictive enrichment will require consideration of patient age and different classification systems for paediatric and adult patients might be required.

Current use of enrichment strategies. As previously noted, the ultimate goal in developing prognostic and predictive enrichment strategies for sepsis is to utilize them in a prospective manner to inform both clinical trial design and treatment decisions. Progress towards this goal will not only require further study and validation of the existing proposed enrichment strategies, but, perhaps more importantly, it will also require the development of rapid assays that are conducive to bedside use in the fast-paced environment of the intensive care unit (ICU). With these limitations in mind, we describe in this section a few examples of enrichment for the purposes of sepsis clinical trial design. Some of these strategies have also been applied to observational data or secondary analyses of previous clinical trials, with existing examples also outlined below.

The first clinical trial to utilize a quasi-predictive enrichment strategy for sepsis used a corticotropin stimulation test (also known as the adrenocorticotropic hormone stimulation test) to identify patients more likely to benefit from corticosteroid therapy ${ }^{45}$. Although the stimulation test results were not used in a prospective manner to guide therapy, in a pre-specified stratified analysis, corticotropin 'non-responders' who received hydrocortisone and fludrocortisone had reduced risk of death, whereas the corticotropin 'responders' did not benefit from hydrocortisone and fludrocortisone. However, these results were not replicated in the follow-up CORTICUS trial ${ }^{45,46}$. In another example, the MONARCHS trial tested the efficacy of an anti-TNF monoclonal antibody in patients with sepsis after enrichment based on blood concentrations of IL-6; patients with a greater degree of systemic inflammation were hypothesized to have higher circulating levels of IL-6 (REF. ${ }^{47}$ ). The monoclonal antibody had no efficacy in the overall cohort or in the subgroup with lower IL-6 concentrations, but the researchers reported a marginal survival benefit among the subgroup with the highest IL- 6 concentrations ${ }^{47}$. The ACROSS trial assessed the ability of acetaminophen (also known as paracetamol) to reduce oxidative injury in patients with severe sepsis. The investigators attempted to enrich the study cohort by selecting patients with detectable plasma cell-free haemoglobin, a marker of oxidative stress. Although acetaminophen did not reduce oxidative injury (as measured by F2-isoprostane levels) at treatment day 3, it did result in lower serum creatinine levels, suggesting that the treatment might have been potentially protective against sepsis-associated $\mathrm{AKI}^{48}$. The EUPHRATES trial used a rapid endotoxin assay to enrol patients with high endotoxin levels for randomization to standard therapy plus polymyxin B haemoperfusion or sham haemoperfusion ${ }^{49}$. Despite the a priori selection of patients with higher endotoxin concentrations, the use of polymyxin B haemoperfusion did not improve survival compared with the sham haemoperfusion group ${ }^{49}$. Finally, the SCARLET trial utilized the international normalized ratio and platelet count - two readily available laboratory tests - to identify patients with sepsis-associated coagulopathy who might benefit from recombinant human thrombomodulin therapy ${ }^{50}$. Unfortunately, despite this pragmatic predictive enrichment approach, treatment with thrombomodulin was not associated with reduced mortality ${ }^{50}$.

These landmark studies represent initial attempts to apply single biomarker-based enrichment strategies to conduct interventional clinical trials in sepsis. Although these single biomarker enrichment strategies are pragmatic and clinically attractive, perhaps a major lesson learned from these studies is that future enrichment strategies for sepsis will need to evolve to capture the biological complexity of sepsis. This improved enrichment will become increasingly important as newer, more invasive and costly therapies are proposed. A pertinent example is extracorporeal cytokine removal, a potential therapy for sepsis currently being studied with some early promise ${ }^{51}$. Given the high cost and invasive nature of this treatment, developing a successful predictive enrichment strategy for its implementation and study will be of the utmost importance. 
With regard to the secondary analysis of existing data, thus far the role of adjunctive corticosteroid use in septic shock is perhaps the question that has been most robustly examined via post-hoc analysis. Unfortunately, several large clinical trials reported competing conclusions regarding the benefit and safety of corticosteroids in septic shock ${ }^{45,46,52-56}$ making it difficult to translate the results of those trials to the bedside. However, subsequent studies examined the role of prognostic and predictive enrichment to retrospectively select subsets of patients more likely to benefit from corticosteroid treatment. In 2014, Funk and colleagues noted in a retrospective analysis of a multicentre study of low-dose corticosteroid administration in sepsis that patients with higher severity of illness scores who received steroids had lower mortality than an unselected group ${ }^{57}$. Another study attempted to utilize a similar prognostic enrichment approach and examined a large heterogeneous group of paediatric patients stratified by PERSEVERE mortality risk, but failed to demonstrate any benefit from treatment with corticosteroids among any of the PERSEVERE-based risk groups ${ }^{58}$. However, in a subsequent study, combining prognostic enrichment (PERSEVERE) with paediatric endotype A or B assignment enabled the retrospective identification of a subset of paediatric patients who seemed to benefit from treatment with corticosteroids ${ }^{59}$. In this study, endotype B patients with an intermediate to high baseline PERSEVERE mortality risk who received corticosteroids had a reduced risk of a complicated disease course compared with the other possible combinations of mortality risk and endotype assignments ${ }^{59}$. Similarly, a secondary analysis of the VANISH clinical trial utilized predictive enrichment to predict corticosteroid responsiveness. In this analysis, randomization to corticosteroids was associated with increased risk of mortality among patients allocated to SRS2 (REF. ${ }^{36}$ ). Although these studies were limited by their post-hoc nature, this strategy of combining prognostic and predictive enrichment to select patients who may benefit from (or be harmed by) corticosteroids is an important model to be replicated in future studies, particularly when considering prospective trial design. Of note, the SHIPSS trial is an ongoing double-blinded, randomized, placebo-controlled trial to test the efficacy of adjunctive hydrocortisone ${ }^{60}$. This trial will include PERSEVERE for prognostic enrichment and endotyping for predictive enrichment. Although these enrichment strategies will not be used to prospectively inform enrolment procedures, they will be used in prespecified post-hoc analyses to validate the existence of a subgroup of children more likely to benefit from adjunctive corticosteroids.

The utilization of prognostic and predictive enrichment in evaluating patients with septic shock has also been applied to other controversial topics and previously failed therapies. For example, the impact of positive fluid balance in critically ill patients, including those with septic shock, is a growing area of research and controversy ${ }^{61}$. Using a prognostic enrichment strategy, a study of a cohort of paediatric patients with septic shock found that positive fluid balance was associated with increased mortality among those with low baseline mortality risk, but not among those with moderate or high risk of mortality on admission ${ }^{62}$. Recombinant human activated protein $\mathrm{C}$, which had been previously associated with reduced mortality but increased bleeding events in patients with severe sepsis ${ }^{63}$, was subsequently shown to be even more beneficial in patients with overt disseminated intravascular coagulation ${ }^{64}$. These findings indicate that identifying patients with DIC might represent a potential predictive enrichment strategy to select patients in whom the potential benefit of the therapy might outweigh the bleeding risk. Unfortunately, attempts at utilization of this enrichment strategy in subsequent prospective trials have failed to demonstrate a mortality benefit from treatment with recombinant human activated protein $C$, again suggesting that the enrichment strategy could not capture the biological complexity of sepsis ${ }^{50,65}$. Similarly, the use of a recombinant IL-1 receptor antagonist, a promising potential therapy given the well-described role of IL-1 in the inflammatory response to sepsis, failed to show a definitive benefit in several clinical trials ${ }^{66-68}$. However, a retrospective subgroup analysis demonstrated that stratification by pre-randomization concentrations of endogenous plasma IL-1 receptor antagonist showed that those with high plasma IL-1 receptor antagonist concentrations appeared to benefit from recombinant IL-1 receptor antagonist therapy ${ }^{69}$. Similarly, the aforementioned EUPHRATES randomized clinical trial found that targeted polymyxin B haemoperfusion for patients with septic shock and high circulating endotoxin levels did not reduce mortality ${ }^{49}$. However, a subsequent post-hoc analysis that excluded patients with extreme levels of endotoxin burden showed that patients in the polymyxin B haemoperfusion group had a significant improvement in mean arterial pressure and an increased number of ventilator-free days, as well as a trend towards decreased mortality, compared with the sham haemoperfusion group ${ }^{70}$. These examples clearly demonstrate the potential benefits of utilizing prognostic and predictive enrichment strategies to reconsider our current understanding of targeted sepsis therapies and to establish a framework from which to continue to develop and improve them.

\section{Enrichment in ARDS}

Although the focus of this Review is precision medicine in sepsis, it is important to highlight that enrichment strategies have also been successfully applied in acute respiratory distress syndrome (ARDS), another heterogeneous critical illness that shares some biological commonalities with and is often caused by sepsis. Calfee and colleagues used plasma biomarkers and latent class analysis to identify two ARDS subphenotypes ${ }^{71,72}$ with potential differential responses to therapy, including fluid management and the use of anti-inflammatory drugs $^{73,74}$. In a subsequent study, significant differences in outcomes were observed between patients with ARDS who were assigned to different paediatric sepsis endotypes, with endotype A patients having significantly higher mortality and a more complicated course of disease than endotype B patients ${ }^{75}$. Given the clinical and biological overlaps between sepsis and ARDS, 
parameters successfully employed in enrichment strategies for patients with ARDS might also be relevant to patients with sepsis and vice versa.

\section{Enrichment in sepsis-associated AKI}

AKI is a common complication of sepsis and substantively contributes to sepsis-related morbidity and mortality. Although data are variable, up to $50 \%$ of all paediatric and adult patients admitted with sepsis are thought to sustain some degree of AKI, a diagnosis that carries an increased risk of poor outcomes, including mortality cited as high as $70 \%$ in some adult populations $^{76-79}$. Unfortunately, although we now recognize the important impact of sepsis-associated AKI, our understanding of its pathophysiology remains incomplete $^{78,80-82}$. Early theories proposed that renal hypoperfusion was the key injury driver; however, a growing body of evidence suggests a considerably more complex process ${ }^{78,80,81}$. The dynamic interplay between the haemodynamic consequences of sepsis (such as decreased cardiac output and systemic vasodilation) and the systemic inflammatory response to infection suggests that sepsis-associated AKI might be caused by a variety of direct and indirect insults as a result of this heterogeneous disease state ${ }^{78,80,81}$. Given its severe consequences, predicting which patients are at highest risk of developing sepsis-associated AKI is clearly important, as it might enable early diagnosis and proactive intervention. Epidemiological studies have identified highrisk populations - including elderly patients, females and patients with baseline comorbid conditions ${ }^{83,84}$ however, specific strategies to predict sepsis-associated AKI at the individual patient level are scarce. The difficulties in elucidating the molecular mechanisms underlying sepsis-associated AKI - and thus in identifying who is at highest risk - are undoubtedly related to the heterogeneity of sepsis itself, as discussed in this Review. Consequently, understanding the molecular signature of ongoing sepsis in an individual patient will likely be crucial to understanding their risk of developing sepsis-associated AKI and its underlying mechanisms.

Predicting sepsis-associated AKI. To date, few successful enrichment strategies have been proposed for the prediction of sepsis-associated AKI. Although the data remain sparse, existing attempts have utilized single biomarkers ${ }^{85-92}$, panels of known biomarkers ${ }^{93}$, microarray for the identification of novel candidate biomarkers ${ }^{94}$ and clinical parameters ${ }^{95-97}$ for AKI prediction and prognostic enrichment.

One of the first candidate biomarkers assessed for its ability to predict sepsis-associated AKI was neutrophil gelatinase-associated lipocalin (NGAL). This protein is a biomarker of renal tubular injury and both serum and urine NGAL have been demonstrated in multiple studies to be highly sensitive but not specific markers of AKI in children with septic shock ${ }^{88,89,92,98}$. Another early candidate serum biomarker was C-terminal afrin fragment, which was shown in one study to be associated with higher incidence of AKI and the need for renal replacement therapy ${ }^{85}$. Several more candidate biomarkers have been subsequently proposed: serum heart-type fatty acid-binding protein $(\mathrm{HFABP})^{89}$, urine soluble triggering receptor expressed on myeloid cells-1 (sTREM-1) ${ }^{86,98}$, kidney injury molecule 1 (KIM-1; also known as hepatitis A virus cellular receptor 1$)^{90}$, netrin-1 $\left(\mathrm{REF}^{9}{ }^{90}\right)$ and the combination of tissue inhibitor of metalloproteinase 2 (TIMP2; also known as metalloproteinase inhibitor 2) and insulin-like growth factorbinding protein 7 (IGFBP7) ${ }^{91}$. These proteins have all been shown to be independent, sensitive markers of sepsis-associated AKI. In particular, urinary netrin-1 and KIM-1 were shown to rise as early as $1 \mathrm{~h}$ after ICU admission, a timeline with promise for enabling early prediction of sepsis-associated $\mathrm{AKI}^{90}$. However, although some of these single and combination biomarker strategies have demonstrated some capability for earlier detection of sepsis-associated AKI, they have not yet provided strategies for prognostic or predictive enrichment for these patients. Given the heterogeneity of sepsis outlined in this Review, a single biomarker strategy alone is unlikely to result in a successful precision medicine approach for sepsis-associated AKI.

Some researchers have also attempted to utilize easily accessible clinical parameters for the early prediction of sepsis-associated AKI. For example, multivariate regression analysis has been used to retrospectively identify independent predictors of sepsis-associated AKI, which included demographic parameters such as age and clinical risk factors such as hypotension ${ }^{97}$. In another study, the combination of high central venous pressure and elevated renal resistive indices, as measured by ultrasound in patients with sepsis, was found to be a sensitive, early predictor of sepsis-associated AKI ${ }^{95}$. More recently, a pilot study combining sequential organ failure assessment scores and biomarker measurements (specifically, serum and urine NGAL) demonstrated the ability to predict AKI and mortality with good sensitivity and specificity ${ }^{96}$. However, as discussed in previous sections, although pragmatic approaches such as these are attractive because of their simplicity, they are unlikely to capture the complexity of the ongoing pathophysiology leading to the development of sepsis-associated AKI.

Recognizing the limitations of the aforementioned strategies, one research group utilized microarray technology to retrospectively identify a panel of novel biomarkers associated with sepsis-associated $\mathrm{AKI}^{94}$. In this study, the researchers identified 21 unique gene probes that were upregulated in patients with sepsisassociated AKI and utilization of these probes was found to predict AKI with high sensitivity and specificity ${ }^{94}$. In particular, serum protein levels of matrix metalloproteinase 8 and elastase 2 (the protein products of two of the 21 candidate genes) had a high sensitivity for AKI prediction, although they demonstrated low specificity $^{94}$. Building upon this work, we developed immunoassays to measure the protein products of five of the previously reported 21 candidate genes - elastase 2 , fibroblast growth factor 13, matrix metalloproteinase 8, olfactomedin 4 and proteinase $3\left(\right.$ REF. $\left.^{93}\right)$. Measurement of these five candidate serum biomarkers enabled CART analysis to determine the probability of AKI on day 3 after presentation with septic shock ${ }^{93}$. The model had 
an excellent capability to predict day $3 \mathrm{AKI}$, with an area under the curve of 0.95 in the derivation cohort and 0.83 in a separate test cohort ${ }^{93}$.

Understanding the variable pathophysiology of sepsis-associated AKI at the individual patient level will be crucial to translating these tools into feasible strategies for prognostic and predictive enrichment. One recent study utilized existing sepsis precision medicine strategies to demonstrate an independent association between allocation to paediatric sepsis endotype A and severe $\mathrm{AKI}^{99}$. Given that patients assigned to sepsis endotype A are characterized by repression of genes associated with the peroxisome proliferator-activated receptor a (PPARa) signalling pathway, the investigators proposed that PPAR $\alpha$-driven fatty acid metabolism was a potential mechanism underlying the association with severe $\mathrm{AKI}^{99}$. This theory is further supported by the fact that PPARa-deficient mice had higher rates of kidney injury than wild-type mice challenged with sepsis; this experimental model might enable future mechanistic studies ${ }^{99}$. This preliminary work provides some insight into potential biologic targets for the understanding and treatment of sepsis-associated AKI, but considerably more work remains to be done. We suggest that similar attempts at leveraging existing sepsis precision medicine research should be undertaken in an effort to develop predictive enrichment strategies for sepsis-associated AKI.

\section{Future directions}

As outlined above, the utilization of discovery-based transcriptomics by multiple research teams has allowed the development of prognostic and predictive enrichment strategies for sepsis. However, further research is required to fully embrace a precision medicine strategy - one in which interventions are targeted in real time based on mortality risk and the biological features of individual patients. Collaborations across investigative teams will need to include efforts to harmonize existing sepsis subgroups, bank specimens and share data, whereas collaborations with industry will be crucial to facilitate the development of rapid and readily available clinical assays. The importance of consensus sepsis subgrouping cannot be overstated and, although some efforts to analyse potential overlap among the four major subclassification systems outlined in this review have been made $23,24,35,37$, the goal of harmonization has not yet been realized. However, as previously mentioned, attempts at gene expression-based subtyping of disease are not limited to sepsis and fields more advanced in their precision medicine approach have achieved this goal of consensus subclassification. A particularly pertinent example is colorectal cancer, a disease that at one time had six independent molecular classification systems that complicated both research and outcome tracking. An international consortium of experts was convened and, through large-scale data-sharing and collaboration, a consensus gene-expression-based subtyping system was developed, which has facilitated new research and allowed standardization of the molecular subclassification of colon cancer ${ }^{100}$. A similar consensus regarding the molecular subtyping of sepsis would be beneficial, but such a system will likely require a similar large-scale international effort, which has not yet been organized.

Post-hoc analyses of clinical trials. Although sepsis genomics are not yet being utilized routinely to inform prospective, or adaptive, clinical trial design, the aforementioned examples of post-hoc analyses of clinical trials $^{36,49,59,62,64,69,70}$ provide a blueprint for researchers on how to leverage our current capabilities. As mentioned, taking advantage of these opportunities to utilize prognostic and predictive enrichment strategies to re-analyse existing data will require sharing and collaboration among investigators. These analyses will not provide definitive results but might provide a rationale for further testing of previously failed therapies and inform future clinical-trial design. Importantly, funding of interventional clinical trials related to such insights should include support for the creation of specimen biobanks that can be used for the development and validation of enrichment strategies. The creation of such biobanks should include the development of protocols for the use and sharing of specimens and data among research groups to maximize their benefits.

Technological limitations. Although genomic technologies have enabled the discoveries presented in this review, the clinical application of these approaches is a major challenge. Unlike the application of genomic technologies for clinical oncology, decision-making among critically ill patients with sepsis must occur within a very short time frame and thus requires actionable data within hours ${ }^{101}$. This obstacle prompted researchers to test the feasibility of using smaller numbers of genes for patient subclassification ${ }^{23,30,31,102}$. These simplified approaches appear to yield reliable classifications, but require further testing. Other promising strategies that might bring this technology to the bedside include the use of protein and metabolomics biomarkers. However, more collaboration with industry is needed to develop technologies with the reliability and timeliness required for use in a critically ill patient.

Another important issue relates to the basic questions of what tissue to test and when. Until now, the vast majority of genomic work in sepsis has focused on peripheral blood leukocytes. This is logical for a variety of reasons, most notably because leukocytes are directly involved in the pathophysiology of sepsis and because access to whole blood (and therefore, leukocytes) is relatively non-invasive and requires relatively minimal processing $^{103-105}$. However, the gene expression patterns of circulating leukocytes might not accurately reflect the pattern of other relevant immune cells that are not practically accessible in critically ill patients (for example, alveolar macrophages, splenic lymphocytes or kidney immune cells) ${ }^{101}$, nor do they provide organ-specific gene expression information. Continued investigation of the biological mechanisms of sepsis will help us to ascertain what cells other than leukocytes might be relevant for analysis in patients with sepsis. An emerging technology that might not only enhance our mechanistic understanding of sepsis but also help to identify potential therapeutic targets is single-cell RNA sequencing. 
This technique provides considerably more detailed information about gene expression at an individual cellular level than analyses based on bulk RNA ${ }^{106}$. A current ambitious and relevant example of how organ-specific information may be utilized is the "Kidney Precision Medicine Project", which is aimed at advancing our understanding of acute and chronic kidney disease by creating a database of kidney biopsy specimens ${ }^{107,108}$. The creation of such a large-scale repository of samples will undoubtedly lead to significant advances in the field, and should be a model to be replicated in other disease processes.

Finally, the question of when to test is also very important. The timing of sepsis onset in a patient cannot be reliably known and experimental studies have demonstrated that genomic changes in response to endotoxin or other forms of inflammation are rapid and dynamic $^{109}$. Therefore, when collecting samples at a particular time point after initial presentation with sepsis, each patient is likely to be at a different stage of sepsis, complicating the interpretation of data. Furthermore, that sample is representative of one particular moment in time, whereas changes in the genomic response to infection of a patient are ongoing. This concept was highlighted by two studies of children with sepsis, the first of which demonstrated significant variability in gene expression at different time points during the evolution of sepsis secondary to meningococcaemia ${ }^{110}$. The second study demonstrated that about one-third of children with septic shock change endotype over the first 3 days of admission ${ }^{34}$. As a result, the utilization of gene expression technology in sepsis will likely require sampling at various time points as a patient's sepsis molecular signature on day 1 may not be the same as on day 3 , a requirement that again highlights the importance of having rapid and cost-effective assays available for clinical use to enable serial measurements. Understanding of the importance of these temporal changes also led to the development of statistical methods to analyse gene expression data over time $\mathrm{e}^{111-114}$, as well as the utilization of dynamic mathematical models of critical illness ${ }^{115,116}$. The Society for Complexity in Acute Illness was formed in 2004 in response to a growing understanding of the dynamic nature of critical illness and the need to develop strategies that enable modelling of changes over time to predict patient trajectories ${ }^{116}$. Although the specifics of these models are outside the scope of this review, this group has provided consensus guidelines for the use of dynamic mathematical modelling for biomedical research ${ }^{116}$. In particular, mechanistic modelling of sepsis molecular signatures might provide an avenue for overcoming the current technological limitations of single time point gene expression approaches (that is, failure to capture dynamic changes of gene expression) alluded to above ${ }^{115}$. Similar to the need for rapid assay development, the evolution of these modelling technologies for real-time interpretation and prediction of changes will be important for the advancement of precision medicine in sepsis.

\section{Conclusions}

Because of its heterogeneity, the management of sepsis at the individual patient level is ideally suited for a precision medicine approach. Although several investigators have successfully utilized leukocyte-derived mRNA and a discovery-based approach to subgroup patients on the basis of biological similarities, the implementation of these prognostic and predictive enrichment strategies at the bedside of patients is limited by several important factors. Further progress is contingent on collaborations between investigators to facilitate a consensus sepsis subclassification, which would enable the large-scale standardization of research efforts. Collaborations with industry are also required to develop technology that is both cost-effective and rapid enough to make its utilization clinically feasible at the bedside of a critically ill patient. Finally, more longitudinal studies of the molecular changes seen over time in sepsis, and how those changes might impact patient outcomes and response to therapies, are required to inform clinicians on when and how to test patients for the purposes of enrichment. As we continue to strive towards these goals, a logical step is to apply our current strategies in post-hoc analyses of previously failed clinical trials, which will again require collaboration and data-sharing among investigators.

The lessons learned from precision medicine research in sepsis are likely to provide important insights into the phenomenon of sepsis-associated AKI, a common and substantial consequence of sepsis. Although research in this area remains limited, it appears that leveraging our understanding of the sepsis molecular signature of an individual might have a key role in predicting their risk of $\mathrm{AKI}$ and its underlying biological mechanisms. Continued research in this area should elucidate the complex pathophysiology of sepsis-associated AKI and facilitate the development and testing of targeted therapies.

Published online 11 September 2019
1. Hotchkiss, R. S. et al. Sepsis and septic shock. Nat. Rev. Dis. Prim. 2, 16045 (2016).

2. Singer, E. et al. Neutrophil gelatinase-associated lipocalin: pathophysiology and clinical applications. Acta Physiol. 207, 663-672 (2013).

3. Marshall, J. C. Why have clinical trials in sepsis failed? Trends Mol. Med. 20, 195-203 (2014).

4. Collins, F. S. \& Varmus, H. A new initiative on precision medicine. N. Engl. J. Med. 372, 793-795 (2015)

5. Wong, H. R. Intensive care medicine in 2050: precision medicine. Intensive Care Med. 43, 1507-1509 (2017).

6. Tsimberidou, A.-M. Initiative for molecular profiling and advanced cancer therapy and challenges in the implementation of precision medicine. Curr. Probl. Cancer 41, 176-181 (2017)
7. Coyne, G. O., Takebe, N. \& Chen, A. P. Defining precision: The precision medicine initiative trials NCI-MPACT and NCI-MATCH. Curr. Probl. Cancer 41, 182-193 (2017)

8. Seibel, N. L. et al. Pediatric oncology enters an era of precision medicine. Curr. Probl. Cancer 41, 194-200 (2017).

9. The White House President Barack Obama. The precision medicine initiative. The White House https://obamawhitehouse.archives.gov/precisionmedicine (2015).

10. Prescott, H. C., Calfee, C. S., Thompson, B. T. Angus, D. C. \& Liu, V. X. Toward smarter lumping and smarter splitting: rethinking strategies for sepsis and acute respiratory distress syndrome clinical trial design. Am. J. Respir. Crit. Care Med. 194, 147-155 (2016).
11. Center for Drug Evaluation and Research. Enrichment strategies for clinical trials to support approval of human drugs and biological products (FDA, 2019).

12. CONSENSUS Trial Study Group. Effects of enalapril on mortality in severe congestive heart failure. results of the cooperative north scandinavian enalapril survival study (CONSENSUS). N. Engl. J. Med. 316 1429-1435 (1987)

13. Piccart-Gebhart, M. J. et al. Trastuzumab after adjuvant chemotherapy in HER2-positive breast cancer. N. Engl. J. Med. 353, 1659-1672 (2005).

14. Zhang, Z., Zhang, G., Goyal, H., Mo, L. \& Hong, Y Identification of subclasses of sepsis that showed different clinical outcomes and responses to amount of fluid resuscitation: a latent profile analysis. Crit. Care 22, 347 (2018) 
15. Gårdlund, B. et al. Six subphenotypes in septic shock: latent class analysis of the PROWESS shock study. J. Crit. Care 47, 70-79 (2018).

16. Bhavani, S. V. et al. Identifying novel sepsis subphenotypes using temperature trajectories. Am J. Respir. Crit. Care Med. 200, 327-335 (2019).

17. Seymour, C. W. et al. Derivation, validation, and potential treatment implications of novel clinical phenotypes for sepsis. JAMA 321, 2003-2017 (2019)

18. Wong, H. R. et al. The pediatric sepsis biomarker risk model. Crit. Care 16, R174 (2012).

19. Wong, H. R. et al. Testing the prognostic accuracy of the updated pediatric sepsis biomarker risk model. PLOS ONE 9, e86242 (2014).

20. Wong, H. R. et al. Pediatric sepsis biomarker risk model-ii: redefining the pediatric sepsis biomarker risk model with septic shock phenotype. Crit. Care Med. 44, 2010-2017 (2016).

21. Wong, H. R. et al. A multibiomarker-based outcome risk stratification model for adult septic shock*. Crit. Care Med. 42, 781-789 (2014).

22. Wong, H. R. et al. Improved risk stratification in pediatric septic shock using both protein and mrna biomarkers. PERSEVERE-XP. Am. J. Respir. Crit. Care Med. 196, 494-501 (2017).

23. Scicluna, B. P. et al. Classification of patients with sepsis according to blood genomic endotype: a prospective cohort study. Lancet Respir. Med. 5 816-826 (2017)

24. Sweeney, T. E. et al. Unsupervised analysis of transcriptomics in bacterial sepsis across multiple datasets reveals three robust clusters. Crit. Care Med. 46, 915-925 (2018)

25. Wong, H. R. et al. Identification of pediatric septic shock subclasses based on genome-wide expression profiling. BMC Med. 7, 34 (2009)

26. Wong, H. R. et al. Toward a clinically feasible gene expression-based subclassification strategy for septic shock: proof of concept. Crit. Care Med. 38 1955-1961 (2010).

27. Wong, H. R. et al. Validation of a gene expressionbased subclassification strategy for pediatric septic shock. Crit. Care Med. 39, 2511-2517 (2011).

28. Wong, H. R. et al. Developing a clinically feasible personalized medicine approach to pediatric septic shock. Am. J. Respir. Crit. Care Med. 191, 309-315 (2015).

29. Langley, R. J. et al. An integrated clinico-metabolomic model improves prediction of death in sepsis. Sci. Transl Med. 5, 195ra95 (2013).

30. Sweeney, T. E. et al. A community approach to mortality prediction in sepsis via gene expression analysis. Nat. Commun. 9, 694 (2018)

31. Davenport, E. E. et al. Genomic landscape of the individual host response and outcomes in sepsis: a prospective cohort study. Lancet Respir. Med. 4 259-271 (2016)

32. Kaplan, J. M. \& Wong, H. R. Biomarker discovery and development in pediatric critical care medicine. Pediatr. Crit. Care Med. 12, 165-173 (2011).

33. Meyer, N. J. et al. A functional synonymous coding variant in the IL1RN gene is associated with survival in septic shock. Am. J. Respir. Crit. Care Med. 190, 656-664 (2014).

34. Wong, H. R. et al. Endotype transitions during the acute phase of pediatric septic shock reflect changing risk and treatment response. Crit. Care Med. 46 e242-e249 (2018)

35. Burnham, K. L. et al. Shared and distinct aspects of the sepsis transcriptomic response to fecal peritonitis and pneumonia. Am. J. Respir. Crit. Care Med. 196 328-339 (2017).

36. Antcliffe, D. B. et al. Transcriptomic signatures in sepsis and a differential response to steroids: from the VANISH randomized trial. Am. J. Respir. Crit. Care Med. 199, 980-986 (2018)

37. Wong, H. R., Sweeney, T. E., Hart, K. W., Khatri, P. $\&$ Lindsell, C. J. Pediatric sepsis endotypes among adults with sepsis. Crit. Care Med. 45, e1289-e1291 (2017).

38. Wynn, J. L. et al. Defective innate immunity predisposes murine neonates to poor sepsis outcome but is reversed by TLR agonists. Blood 112, 1750-1758 (2008).

39. Wynn, J. L. et al. The influence of developmental age on the early transcriptomic response of children with septic shock. Mol. Med. 17, 1146-1156 (2011).

40. Wynn, J. L. et al. Postnatal age is a critical determinant of the neonatal host response to sepsis. Mol. Med. 21, 496-504 (2015).
41. Raymond, S. L. et al. Unique transcriptomic response to sepsis is observed among patients of different age groups. PLOS ONE 12, e0184159 (2017).

42. Atkinson, S. J. et al. Matrix metalloproteinase-8 augments bacterial clearance in a juvenile sepsis model. Mol. Med. 22, 455-463 (2016).

43. Cernada, M. et al. Genome-wide expression profiles in very low birth weight infants with neonatal sepsis. Pediatrics 133, e1203-e1211 (2014).

44. Gentile, L. F. et al. Protective immunity and defects in the neonatal and elderly immune response to sepsis. J. Immunol. 192, 3156-3165 (2014).

45. Annane, D. et al. Effect of treatment with low doses of hydrocortisone and fludrocortisone on mortality in patients with septic shock. JAMA 288, 862-871 (2002).

46. Sprung, C. L. et al. Hydrocortisone therapy for patients with septic shock. N. Engl. J. Med. 358 , 111-124 (2008)

47. Panacek, E. A. et al. Efficacy and safety of the monoclonal anti-tumor necrosis factor antibody F(ab')2 fragment afelimomab in patients with severe sepsis and elevated interleukin-6 levels. Crit. Care Med. 32, 2173-2182 (2004).

48. Janz, D. R. et al. Randomized, placebo-controlled trial of acetaminophen for the reduction of oxidative injury in severe sepsis: the acetaminophen for the reduction of oxidative injury in severe sepsis trial. Crit. Care Med. 43, 534-541 (2015)

49. Dellinger, R. P. et al. Effect of targeted polymyxin b hemoperfusion on 28-day mortality in patients with septic shock and elevated endotoxin level: the EUPHRATES randomized clinical trial. JAMA 320 1455-1463 (2018).

50. Vincent, J.-L. et al. Effect of a recombinant human soluble thrombomodulin on mortality in patients with sepsis-associated coagulopathy: the SCARLET randomized clinical trial. JAMA 321, 1993-2002 (2019).

51. Hawchar, F. et al. Extracorporeal cytokine adsorption in septic shock: a proof of concept randomized, controlled pilot study. J. Crit Care 49, 172-178 (2019).

52. Annane, D. et al. Hydrocortisone plus fludrocortisone for adults with septic shock. N. Engl. J. Med. 378 809-818 (2018)

53. Venkatesh, B. et al. Adjunctive glucocorticoid therapy in patients with septic shock. N. Engl. J. Med. 378 797-808 (2018).

54. Casserly, B. et al. Low-dose steroids in adult septic shock: results of the surviving sepsis campaign. Intensive Care Med. 38, 1946-1954 (2012).

55. Menon, S. et al. Urinary biomarker incorporation into the renal angina index early in intensive care unit admission optimizes acute kidney injury prediction in critically ill children: a prospective cohort study. Nephrol. Dial. Transplant. 31, 586-594 (2016).

56. Zimmerman, J. J. \& Williams, M. D. Adjunctive corticosteroid therapy in pediatric severe sepsis: observations from the RESOLVE study. Pediatr Crit. Care Med. 12, 2-8 (2011).

57. Funk, D. et al. Low-dose corticosteroid treatment in septic shock: a propensity-matching study. Crit. Care Med. 42, 2333-2341 (2014).

58. Atkinson, S. J. et al. Corticosteroids and pediatric septic shock outcomes: a risk stratified analysis. PLOS ONE 9, e112702 (2014).

59. Wong, H. R. et al. Combining prognostic and predictive enrichment strategies to identify children with septi shock responsive to corticosteroids. Crit. Care Med. 44, e 1000-e1003 (2016)

60. US National Library of Medicine. ClinicalTrials.gov https://clinicaltrials.gov/ct2/show/NCT03401398 (2019).

61. Alobaidi, R. et al. Association between fluid balance and outcomes in critically ill children. JAMA Pediatr. 172, 257-268 (2018).

62. Abulebda, K. et al. Post-ICU admission fluid balance and pediatric septic shock outcomes: a risk-stratified analysis. Crit. Care Med. 42, 397-403 (2014).

63. Bernard, G. R. et al. Efficacy and safety of recombinant human activated protein $C$ for severe sepsis. N. Engl. J. Med. 344, 699-709 (2001)

64. Dhainaut, J.-F. et al. Treatment effects of drotrecogin alfa (activated) in patients with severe sepsis with or without overt disseminated intravascular coagulation. J. Thromb. Haemost. 2, 1924-1933 (2004).

65. Vincent, J.-L. et al. A randomized, double-blind, placebo-controlled, Phase $2 \mathrm{~b}$ study to evaluate the safety and efficacy of recombinant human soluble thrombomodulin, ART-123, in patients with sepsis and suspected disseminated intravascular coagulation. Crit. Care Med. 41, 2069-2079 (2013)

66. Fisher, C. J. et al. Initial evaluation of human recombinant interleukin-1 receptor antagonist in the treatment of sepsis syndrome: a randomized, open-label, placebo-controlled multicenter trial. Crit. Care Med. 22, 12-21 (1994).

67. Fisher, C. J. et al. Recombinant human interleukin receptor antagonist in the treatment of patients with sepsis syndrome. Results from a randomized, double-blind, placebo-controlled trial. Phase III rhIL-1 ra Sepsis Syndrome Study Group. JAMA 271 1836-1843 (1994).

68. Opal, S. M. et al. Confirmatory interleukin-1 receptor antagonist trial in severe sepsis: a phase III, randomized, double-blind, placebo-controlled, multicenter trial. The Interleukin-1 Receptor Antagonist Sepsis Investigator Group. Crit. Care Med. 25, 1115-1124 (1997)

69. Meyer, N. J. et al. Mortality benefit of recombinant human interleukin-1 receptor antagonist for sepsis varies by initial interleukin- 1 receptor antagonist plasma concentration. Crit. Care Med. 46, 21-28 (2018).

70. Klein, D. J. et al. Polymyxin B hemoperfusion in endotoxemic septic shock patients without extreme endotoxemia: a post hoc analysis of the EUPHRATES trial. Intensive Care Med. 44, 2205-2212 (2018).

71. Calfee, C. S. et al. Subphenotypes in acute respiratory distress syndrome: latent class analysis of data from two randomised controlled trials. Lancet Respir. Med. 2, 611-620 (2014).

72. Delucchi, K. et al. Stability of ARDS subphenotypes over time in two randomised controlled trials. Thorax 73, 439-445 (2018).

73. Calfee, C. S. et al. Acute respiratory distress syndrome subphenotypes and differential response to simvastatin: secondary analysis of a randomised controlled trial. Lancet Respir. Med. 6, 691-698 (2018).

74. Famous, K. R. et al. Acute respiratory distress syndrome subphenotypes respond differently to randomized fluid management strategy. Am. J. Respir. Crit. Care Med. 195, 331-338 (2017).

75. Yehya, N., Thomas, N. J. \& Wong, H. R. Evidence of endotypes in pediatric acute hypoxemic respiratory failure caused by sepsis. Pediatr. Crit. Care Med. 20 . 110-112 (2019).

76. Uchino, S. et al. Acute renal failure in critically ill patients: a multinational, multicenter Study. JAMA 294, 813-818 (2005).

77. Bouchard, J. et al. A Prospective international multicenter study of AKI in the intensive Care Unit. Clin. J. Am. Soc. Nephrol. 10, 1324-1331 (2015).

78. Alobaidi, R., Basu, R. K., Goldstein, S. L. \& Bagshaw, S. M. Sepsis-associated acute kidney injury. Semin. Nephrol. 35, 2-11 (2015).

79. Bagshaw, S. M. et al. Acute kidney injury in septic shock: clinical outcomes and impact of duration of hypotension prior to initiation of antimicrobial therapy. Intensive Care Med. 35, 871-881 (2009).

80. Kellum, J. A. \& Prowle, J. R. Paradigms of acute kidney injury in the intensive care setting. Nat. Rev. Nephrol. 14, 217 (2018)

81. Gomez, H. et al. A unified theory of sepsis-induced acute kidney injury: inflammation, microcirculatory dysfunction, bioenergetics, and the tubular cell adaptation to injury. Shock 41, 3-11 (2014).

82. Schrier, R. W. \& Wang, W. Acute renal failure and sepsis. N. Engl. J. Med. 351, 159-169 (2004).

83. Bagshaw, S. M. et al. Septic acute kidney injury in critically ill patients: clinical characteristics and outcomes. Clin. J. Am. Soc. Nephrol. 2, 431-439 (2007).

84. Bagshaw, S. M., George, C., Bellomo, R. \& ANZICS Database Management Committee. Early acute kidney injury and sepsis: a multicentre evaluation. Crit. Care 12, R47 (2008).

85. Drey, M. et al. C-terminal agrin fragment (CAF) reflects renal function in patients suffering from severe sepsis or septic shock. Clin. Lab. 61, 69-76 (2015)

86. Yuan, Z. K. et al. [Value of urine soluble triggering receptor expressed on myeloid cells-1 in the early diagnosis of sepsis associated acute kidney injury]. Zhonghua Er Ke Za Zhi 56, 342-346 (2018).

87. Kim, J. H. et al. Delta neutrophil index for the prediction of the development of sepsis-induced acute kidney injury in the emergency department. Shock https://doi.org/10.1097/SHK.0000000000001299 (2019).

88. Wheeler, D. S. et al. Serum neutrophil gelatinase associated lipocalin (NGAL) as a marker of acute 
kidney injury in critically ill children with septic shock. Crit. Care Med. 36, 1297-1303 (2008)

89. Jiang, D. et al. Serum heart-type fatty acid-binding protein as a predictor for the development of sepsisassociated acute kidney injury. Expert Rev. Mol. Diagn. 17, 1-9 (2019).

90. Tu, Y. et al. Urinary netrin-1 and KIM-1 as early biomarkers for septic acute kidney injury. Ren. Fail. 36, 1559-1563 (2014)

91. Honore, P. M. et al. Urinary tissue inhibitor of metalloproteinase- 2 and insulin-like growth factorbinding protein 7 for risk stratification of acute kidney injury in patients with sepsis. Crit. Care Med. 44, 1851-1860 (2016)

92. Kim, S. et al. Is plasma neutrophil gelatinase-associated lipocalin a predictive biomarker for acute kidney injury in sepsis patients? A systematic review and meta-analysis. J. Crit. Care 33, 213-223 (2016).

93. Wong, H. R. et al. A multibiomarker-based model for estimating the risk of septic acute kidney injury. Crit. Care Med. 43, 1646-1653 (2015).

94. Basu, R. K et al. Identification of candidate serum biomarkers for severe septic shock-associated kidney injury via microarray. Crit. Care 15, R273 (2011).

95. Song, J. et al. Value of the combination of renal resistance index and central venous pressure in the early prediction of sepsis-induced acute kidney injury. J. Crit. Care 45, 204-208 (2018)

96. Lee, C.-W. et al. A combination of SOFA score and biomarkers gives a better prediction of septic AKI and in-hospital mortality in critically ill surgical patients: a pilot study. World J. Emerg. Surg. 13, 41 (2018)

97. Zhou, J. et al. A simple risk score for prediction of sepsis associated-acute kidney injury in critically ill patients. J. Nephrol. https://doi.org/10.1007/s40620 019-00625-y (2019).

98. Dai, X. et al. Diagnostic value of neutrophil gelatinaseassociated lipocalin, cystatin C, and soluble triggering receptor expressed on myeloid cells- 1 in critically ill patients with sepsis-associated acute kidney injury. Crit. Care 19, 223 (2015).

99. Iwaki, T. et al. PPAR a contributes to protection against metabolic and inflammatory derangements associated with acute kidney injury in experimental sepsis. Physiol. Rep. 7, e14078 (2019).

100. Guinney, J. et al. The consensus molecular subtypes of colorectal cancer. Nat. Med. 21, 1350-1356 (2015).

101. Maslove, D. M. $\&$ Wong, H. R. Gene expression profiling in sepsis: timing, tissue, and translational considerations. Trends Mol. Med. 20, 204-213 (2014).

102. Wong, H. R., Sweeney, T. E. \& Lindsell, C. J Simplification of a septic shock endotyping strategy for clinical application. Am. J. Respir. Crit. Care Med. 195, 263-265 (2017).

103. Tang, B. M. P., McLean, A. S., Dawes, I. W., Huang, S. J. $\&$ Lin, R. C. Y. Gene-expression profiling of peripheral blood mononuclear cells in sepsis. Crit. Care Med. 37 882-888 (2009).

104. Tang, B. M. P., McLean, A. S., Dawes, I. W., Huang, S. J. $\&$ Lin, R. C. Y. The use of gene-expression profiling to identify candidate genes in human sepsis. Am. J. Respir Crit. Care Med. 176, 676-684 (2007).

105. Vartanian, K. et al. Gene expression profiling of whole blood: comparison of target preparation methods for accurate and reproducible microarray analysis. BMC Genomics 10, 2 (2009).

106. Hedlund, E. \& Deng, Q. Single-cell RNA sequencing: technical advancements and biological applications. Mol. Aspects Med. 59, 36-46 (2018).

107. National Institute of Diabetes and Digestive and Kidney Diseases. Kidney precision medicine project NIDDK, 2018)

108. Kidney Precision Medicine Project. Changing the way we understand and treat kidney disease. KPMP https://kpmp.org/ (2019)

109. Talwar, S. et al. Gene expression profiles of peripheral blood leukocytes after endotoxin challenge in humans. Physiol. Genomics 25, 203-215 (2006).

110. Kwan, A., Hubank, M., Rashid, A., Klein, N. \& Peters, M. J. Transcriptional instability during evolving sepsis may limit biomarker based risk stratification. PLOS ONE 8, e60501 (2013).

111. McDunn, J. E. et al. Plasticity of the systemic inflammatory response to acute infection during critical illness: development of the riboleukogram. PLOS ONE 3, e 1564 (2008).
112. Polpitiya, A. D., McDunn, J. E., Burykin, A., Ghosh, B. K $\&$ Cobb, J. P. Using systems biology to simplify complex disease: immune cartography. Crit. Care Med. 37 , S16-S21 (2009)

113. Zhou, B. et al. Analysis of factorial time-course microarrays with application to a clinical study of burn injury. Proc. Natl. Acad. Sci. USA 107, 9923-9928 (2010).

114. Storey, J. D., Xiao, W., Leek, J. T., Tompkins, R. G. \& Davis, R. W. Significance analysis of time course microarray experiments. Proc. Natl. Acad. Sci. USA 102, 12837-12842 (2005)

115. Aerts, J.-M., Haddad, W. M., An, G. \& Vodovotz, Y. From data patterns to mechanistic models in acute critical illness. J. Crit. Care 29, 604-610 (2014).

116. Vodovotz, Y. et al. Evidence-based modeling of critical illness: an initial consensus from the society for complexity in acute illness. J. Crit. Care 22, 77-84 (2007).

\section{Author contributions}

H.R.W. and N.L.S, researched the existing literature, discussed the article's content, wrote the text and reviewed or edited the article before submission.

\section{Competing interests}

H.R.W. and the Cincinnati Children's Hospital Research Foundation hold United States Patents for the PERSEVERE biomarkers and paediatric endotyping strategies described in the manuscript. N.L.S. declares no competing interests.

\section{Peer review information}

Nature Reviews Nephrology thanks J. -L. Vincent, Y. Vodovotz, A. Bihorac and the other, anonymous, reviewer(s) for their contribution to the peer review of this work.

\section{Publisher's note}

Springer Nature remains neutral with regard to jurisdictional claims in published maps and institutional affiliations.

\section{RELATED LINKS}

Ingenuity Pathway Analysis legend: http://qiagen.force.com/ KnowledgeBase/articles/Basic_Technical_Q_A/Legend 